\title{
EXPLORING THE KNOWLEDGE AND PRACTICES OF AUSTRALIAN MEDICAL STUDENTS REGARDING INFECTION PREVENTION AND CONTROL IN HOSPITALS: A PILOT STUDY
}

\author{
Maha Pervaz Iqbal, Kerry Uebel, Md. Saiful Islam, Victoria Jabbour, John Hall, Abrar A. Chughtai, Najm Us Saqib Khan*
}

School of Population Health, UNSW Sydney, Australia, *Bahria University Medical and Dental College, Karachi Pakistan

\section{ABSTRACT}

Objective: To assess the knowledge and practice of medical students regarding standard precautions in an Australian undergraduate medical programme.

Study Design: Cross sectional study.

Place and Duration of Study: University of New South Wales, Australia, duration of study was six-years.

Methodology: Fifty medical students who were involved in clinical practice, were invited to complete a comprehensive questionnaire on standard precautions.

Results: The majority of participants agreed that hand hygiene was the most important factor in infection control. Only $32(16 \%)$ knew to use a full personal protective equipment for Ebola. Regarding sharps disposal, 46 (92\%) students always disposed used needles in the recommended bin, 27 (54\%) indicated they sometimes recapped used needle while $25(20 \%)$ sometimes bent used needles.

Conclusion: This study showed that there is a gap between knowledge and practice of medical students regarding hand hygiene and other infection control measures. There is a need for standardized and regular student training in the use of standard precautions in infection prevention and control.

Keywords: Care of medical students, Hand hygiene, Knowledge and practice, Personal protective equipment, Student, Sharps injury and disposal.

How to Cite This Article: Iqbal MP, Uebel K, Islam MS, Jabbour V, Hall J, Chughtai AA, Khan NUS. Exploring the Knowledge and Practices of Australian Medical Students Regarding Infection Prevention and Control in Hospitals: A Pilot Study. Pak Armed Forces Med J 2021; 71(6): $2236-2241$.

This is an Open Access article distributed under the terms of the Creative Commons Attribution License (https://creativecommons.org/licenses/by-nc/4.0/), which permits unrestricted use, distribution, and reproduction in any medium, provided the original work is properly cited.

\section{INTRODUCTION}

In 2007, the World Health Organization (WHO) issued checklists for standard precautions to prevent the transmission of infectious agents in health settings and in 2009, comprehensive guidelines were issued for hand hygiene as one of the basic essentials in infection control. ${ }^{1}$ Standard precautions are considered to be critical infection control practices in health care. Standard precautions include hand hygiene, respiratory hygiene (cough etiquette), routine environmental cleaning, use of personal protective equipment (PPE), waste management and general risk assessment and risk reduction against the transmission of blood-borne or other pathogens (including sharps disposal and injury and selfprotection). ${ }^{2}$ Improving just basic hand hygiene in health care settings has been shown to decrease hospital acquired infections. ${ }^{3}$

Despite these recommendations and the evidence, a systematic review of studies has shown suboptimal compliance with the application of standard precautions in health care settings. ${ }^{4}$ Of concern, is the evidence that doctors have sub-optimal adherence with

Correspondence: Dr Maha Pervaz Iqbal, School of Population Health, UNSW Medicine, UNSW Sydney, Sydney 2052, Australia

Received: 11 Jan 2021; revision received: 17 Sep 2021; accepted: 10 Oct 2021 standard precautions. In a study from Iran, $80 \%$ of doctors reported they had not been trained in standard precautions, and perceived that their knowledge was good but their practices were poor. ${ }^{5}$ In a Swiss study on adherence to hand washing, one of the most basic tenets of infection control, only $57 \%$ of hospital based physicians were observed to be compliant with recommendations. ${ }^{6}$

There have been similar reports of gaps in training, knowledge and practice amongst medical students. A survey of medical students in Saudi Arabia reported that only $26 \%$ had an acceptable knowledge of standard precautions and students reported that their sources of knowledge were self-learning and bed side informal practice and that they had insufficient formal training. ${ }^{7}$ Surveys of health care students in France and Italy showed that although knowledge of standard precautions was good, many students did not know that the major source of nosocomial infections was via the hands of health care workers. ${ }^{8,9}$ These gaps in knowledge are widespread with reports of poor knowledge and practice in medical students in the United Kingdom (UK) 10 and Saudi Arabia. ${ }^{7}$

There is limited literature on the knowledge and practice of standard precautions of medical students in 
Australia. The aim of this pilot study was to assess the knowledge and practice of medical students regarding standard precautions which include hand hygiene, use of personal protective equipment, sharps disposal and injuries and protection of medical students in an Australian undergraduate medical programme.

\section{METHODOLOGY}

This study was undertaken at an Australian Medical School-University of New South Wales. The medical program was a six-years, integrated course and students had an early introduction to patient contact and clinical experience in teaching hospitals and community settings.

Inclusion Criteria: Undergraduate medical students from Australian Medical School-University of New South Wales were included.

Exclusion Criteria: Non consenting students were excluded.

This pilot study was conducted with a sample of 50 students. An email was sent to all medical students from phase 2 (year 3 and 4) $(n=541)$ and phase 3 (year 5 and 6) $(n=546)$ inviting them to participate and email their expression of interest to the research team. The first fifty students who responded were then invited to complete a semi-structured, paper-based questionnaire which took approximately 30 minutes to complete in a face to face setting.

A comprehensive questionnaire was designed by the authors of this study based on previously validated questionnaires which were used for exploring medical students' knowledge and practice of infection control. The designed questionnaire consisted of both closed and open-ended questions on standard precautions including broad questions about infection prevention and control and the role of students in preventing infections and specific questions about hand hygiene, use of personal protective equipment, sharps disposal and injury and protection of medical students. Questions were designed to explore the knowledge, practice and source of knowledge. In this study, the focus will be on reporting the knowledge and practice only. Basic demographic information was asked about the year of study, gender, days spent in the clinical workplace and approximate number of patients seen per week.

Data were entered and analysed by Statistical Package for Social Sciences (SPSS) version 23. Rates and frequencies were calculated for various variables. A chi-square test was performed for categorical variables. The primary author reviewed the qualitative data thoroughly and then categorised the data under different themes and sub-themes. The themes and subthemes were presented and confirmed among the research team and any discrepancy was resolved via discussion. The knowledge question asked students to outline the correct method of hand washing. The answers to this question were checked to analyse whether students had explained the correct technique for hand washing as stated in the WHO hand hygiene guidelines. ${ }^{1}$

\section{RESULTS}

Email invitation was sent to 1087 students and the first 50 students $(4.6 \%)$, who responded, were invited to complete the questionnaire and were included in the study. The distribution of the respondents was as follows: 29 (58\%) were in phase 2 (years 3 and 4) and $21(42 \%)$ in phase 3 (years 5 and 6).

In the broad questions on standard precautions, the majority of students indicated that hand hygiene was the most important factor in preventing patients from acquiring an infection whilst in hospital (example quote below). Some students mentioned the use of PPE and aseptic techniques, and a few students mentioned quarantine and isolation of sick patients as measures of standard precautions. All the students agreed that they had an important role in preventing the spread of infection to patients and that they should practice hand hygiene and follow infection control protocols.

Almost all students 49 (98\%) agreed that hand hygiene reduces infection transfer to patients and health care workers and needs to be practiced. However, their account of their own practice varied. Only $39(78 \%)$ students indicated that they always practiced hand hygiene after patient contact and $30(60 \%)$ before patient contact. For phase 2 students, 22/30 (73\%) reported that they always washed their hand before patient contact whereas, only 8/20 $20(40 \%)$ of the phase 3 students reported always washing hands before patient contact and this difference was statistically significant $(p=0.018)$ as shown in Table-I.

All the students indicated they always practiced hand hygiene after contact with body fluids, 45 (90\%) before conducting an aseptic procedure and only 18 $(36 \%)$ after removal of gloves. Students were asked to list reasons for not washing hands after patient contact and they could indicate more than one option. Many of the students, $42(84 \%)$, who did not always wash hands, indicated that they used the alcohol-based gel instead, but some also indicated that at times they were busy, or forgot being $16(32 \%)$ and $6(12 \%)$ 
respectively. In the questions enquiring about students' observations of hand hygiene practiced in the hospitals, students commented that the easy availability of alcohol hand rub encouraged good practice. Many commented that compliance was not ideal and that doctors were less compliant than nurses and that some senior doctors were not practicing hand hygiene all the time.

Some students commented that they had seniors who encouraged and practiced good hand hygiene, and this influenced the students to comply as well.

Students were asked to indicate which PPE to use for patients with various infectious diseases. Their answers about the of PPE for respiratory infection were presented in Table-II. Two-thirds i.e., 34 (68\%) students enza, while $41(82 \%)$ indicated using a gown as PPE for patients with TB. However, many students indicated that they had not seen these diseases and up to 10 $(20 \%)$ indicated that had no knowledge of which PPE was appropriate to use in each disease.

For patients with Ebola only $35(70 \%)$ indicated they would use gloves and only $8(16 \%)$ understood that full body protection was needed (i.e., gloves, facemask, respirator, gown/overall, goggles, shoe cover and surgical hood), while $11(22 \%)$ students did not know which PPE to use for patients with Ebola. In patients with Multi-Resistant Staphylococcus Aureus (MRSA) only $44(88 \%)$ indicated they would use gloves, $29(58 \%)$ would use a face mask and 41 (82\%) would use a gown.

Table-I: Comparison of standard precaution behaviours between phase 2 and Phase 3 medical students.

\begin{tabular}{l|c|c|c}
\hline Parameters & $\begin{array}{c}\text { Phase-2 } \\
\text { 30 Students, (n)\% }\end{array}$ & $\begin{array}{c}\text { Phase-3 } \\
\text { 20 Students, (n)\% }\end{array}$ & $\begin{array}{c}p \text { - } \\
\text { value }\end{array}$ \\
\hline I always wash my hands before contact with patient & $22(73)$ & $8(40)$ & 0.018 \\
\hline I always wash my hands after contact with patient & $25(83)$ & $14(70)$ & 0.265 \\
\hline I always dispose used syringes in the sharps container & $26(86)$ & $20(100)$ & 0.089 \\
\hline I never recap used needles to prevent injuries & $15(86)$ & $8(40)$ & 0.487 \\
\hline I never bend used needles to prevent injuries & $22(73)$ & $18(90)$ & 0.148 \\
\hline I would always report a sharps injury to my supervisor & $25(83)$ & $219(95)$ & 0.214 \\
\hline
\end{tabular}

Table-II: Students indication of using the PPE for respiratory infections.

\begin{tabular}{|c|c|c|c|c|c|c|c|}
\hline & $\begin{array}{l}\text { Gloves } \\
\text { n (\%) }\end{array}$ & $\begin{array}{c}\text { Face Mask } \\
\text { n }(\%)\end{array}$ & $\begin{array}{l}\text { Respirator } \\
\text { n (\%) }\end{array}$ & $\begin{array}{l}\text { Gown } \\
\text { n (\%) }\end{array}$ & $\begin{array}{l}\text { Goggles or Face } \\
\text { Shield, } n(\%)\end{array}$ & $\begin{array}{c}\text { Shoe Cover } \\
\mathrm{n}(\%)\end{array}$ & $\begin{array}{c}\text { Surgical } \\
\text { Hood/Cap, } \mathrm{n}(\%)\end{array}$ \\
\hline Seasonal influenza & $36(72 \%)$ & $42(84 \%)$ & $8(16 \%)$ & $18(36 \%)$ & $3(6 \%)$ & $1(2 \%)$ & $1(2 \%)$ \\
\hline Avian influenza & $31(62 \%)$ & $28(56 \%)$ & $16(32 \%)$ & $19(38 \%)$ & $8(16 \%)$ & $4(8 \%)$ & $1(2 \%)$ \\
\hline $\begin{array}{l}\text { Pandemic influenza } \\
\text { (due to new strain) }\end{array}$ & $37(74 \%)$ & $35(70 \%)$ & $23(46 \%)$ & $29(58 \%)$ & $10(20 \%)$ & $3(6 \%)$ & $1(2 \%)$ \\
\hline $\begin{array}{l}\text { Severe acute respiratory } \\
\text { syndrome (SARS) }\end{array}$ & $34(68 \%)$ & $30(60 \%)$ & $26(52 \%)$ & $27(54 \%)$ & $14(28 \%)$ & $7(14 \%)$ & $3(6 \%)$ \\
\hline $\begin{array}{l}\text { Middle East Respiratory } \\
\text { Syndrome (MERS) }\end{array}$ & $31(62 \%)$ & $26(52 \%)$ & $24(48 \%)$ & $24(48 \%)$ & $10(20 \%)$ & $5(10 \%)$ & $3(6 \%)$ \\
\hline Tuberculosis (TB) & $44(88 \%)$ & $34(68 \%)$ & $34(68 \%)$ & $41(82 \%)$ & $22(44 \%)$ & $8(16 \%)$ & $3(6 \%)$ \\
\hline
\end{tabular}

indicated that they would use a respirator when in contact with patients with tuberculosis, while the rest indicated they would use a face mask. For patients with any of the six respiratory viruses included in the questionnaire: seasonal influenza, avian influenza, pandemic influenza, severe acute respiratory syndrome (SARS), Middle East Respiratory Syndrome (MERS) and Tuberculosis (TB) 44 (88\%) indicated they would use some respiratory protection, with most $(52 \%-84 \%)$ favouring masks but between $23(46 \%)$ and $27(54 \%)$ indicated using respirators for SARS, MERS and pandemic influenza. Regarding the need for gowns with these respiratory infections between $38-58 \%$ of students indicated that one should use a gown for patients with SARS, avian influenza and pandemic influ-
The majority of students, 42 (92\%) indicated that they always disposed used syringes in the sharps' disposal container and $44(88 \%)$ students indicated that they always report a sharps injury to their supervisor. However, $27(54 \%)$ students indicated that they sometimes recapped needles after use and $10(20 \%)$ that they sometimes bent used needles to prevent injuries. The number of students recapping needles reduced as they progressed in medical school. The majority of students reported that the health care staff were compliant with the sharps' disposal protocol. However, some commented on discrepancies between what was being taught and what was practiced.

Almost all students, 49 (98\%), were aware that they should receive all immunizations before commen- 
cing clinical work. The majority of students had received the varicella vaccine $49(98 \%)$ and annual influenza vaccine, $48(96 \%)$, while two third $34(68 \%$;) had received BCG (Bacillus Calmette-Guérin, TB vaccine). Regarding medical students' knowledge about protection from $\mathrm{TB}, 16(32 \%)$ students were unsure if they should have a tuberculin test after exposure to TB, while only $32(64 \%)$ students were aware that an annual TB test is not indicated for all students and 16 $(32 \%)$ were unsure.

\section{DISCUSSION}

In this pilot study we explored the knowledge and practices of a group of Australian medical students regarding infection prevention and control in clinical settings. The majority of the students demonstrated a clear understanding of the importance of hand hygiene, sharps disposal and injuries and protection of medical students. However, students' practices of these three aspects varied and there were gaps in the students' knowledge and practice of personal protective equipment.

Clearly there is a need for better education on standard precautions amongst health care students but also information on how to link knowledge with practice. ${ }^{11}$ A survey of UK and Irish medical schools' curricula showed that the vast majority were teaching about hospital acquired infection control but less than two thirds were assessing the use of standard precautions and quality and control measures. ${ }^{12}$ A similar survey of 17 Australian medical schools regarding teaching about hand hygiene found that they were using skills stations and case scenarios or lectures to teach students and were using practical exams and competency checks to assess skills ${ }^{13}$. However, this study also identified poor attitudes to hand washing from senior clinicians and negative student attitudes towards the teaching of hand hygiene. ${ }^{13}$ Early interventions in the medical course on hand hygiene showed significant and sustained improvement in student knowledge.

At present, only one study has been published on the practice of hand hygiene amongst medical students in Australia and it identified the need to promote the learning of hand hygiene in students. ${ }^{14}$

Although almost all the students knew of the importance and necessity of hand hygiene in infection control, there were two major gaps. The first was that the majority did not describe using alcohol hand rub as the recommended method for hand hygiene. Alcoholbased hand rub is the gold standard of care for hand hygiene in most clinical contexts, while washing of hands is recommended for situations when the hands are visibly soiled, or if gloves have not been worn in the care of a patient with Clostridium difficile. ${ }^{15,16}$ The second major gap between knowledge and practice, is that only $78 \%$ students indicate that they always use hand hygiene after patient contact and $60 \%$ before patient contact. Comments from students in this study indicate that practice in the hospitals, especially observing their senior doctors' practice, is not ideal. This is similar to findings from many other studies amongst medical students, and observations that senior staff who should be key models of best practice are not always complying. ${ }^{17}$ A study conducted by Barroso et $a l ., 18$ at the Stanford University School of Medicine, found that medical student knowledge was not a significant predictor of behaviour, but a working gel dispenser and observing attending physicians with good hand hygiene practices were reported as the most effective strategies in influencing trainees. Therefore, seniors' role modelling the optimal infection control prevention strategies is important for students to observe to encourage effective practice.

There were also gaps in the students' knowledge of the use of PPE. Although the majority of students knew how to use respiratory protection in the form of face mask or respirator for various respiratory viruses and $\mathrm{TB}$, up to $20 \%$ indicated they did not know which respiratory protection to use. Medical or surgical face masks are used in healthcare settings to protect the wearer from splash and spray of blood and body fluid and to protect patients from transmission of respiratory infections from the health worker. They are also used by sick patients to prevent spread of infection. Medical or surgical masks do not fit to the face and they do not provide adequate protection against small airborne respiratory particles. In contrast to this, N95 respirators are designed for respiratory protection and are used to protect the wearer from small airborne particles. Filtration capacity of respirators is also reported to be higher compared to medical or surgical masks. ${ }^{19}$ The knowledge of appropriate PPE is critical for medical students in relation to respiratory infections especially at this time of pandemic infections like COVID-19. For the current COVID-19 pandemic the recommended PPE involves donning gown, gloves, eye protection (goggles or face shield) and a P2/N95 respirator, which should be fit-checked. ${ }^{20}$ For patients with $\mathrm{TB}$, only $68 \%$ knew that the appropriate respiratory protection for health workers was to use a respirator. The recommendation is 'to wear a correctly fitted $\mathrm{P} 2 / \mathrm{N} 95$ respirator prior to entering the patient-care 
area when an airborne transmissible infectious agent is known or suspected'. ${ }^{21}$ This gap in knowledge may be due to the low incidence of TB in Australia and no previous experience with patients with TB. However, given the world-wide prevalence of $\mathrm{TB}^{22}$ this is important for students to be aware of. Although students had some understanding of respiratory protection for airborne diseases, they appeared to have poor understanding of the indications for using gowns. Some students indicated that gowns were appropriate PPE for both respiratory viruses and TB. The use of gowns is not recommended for either TB or the five viruses mentioned. ${ }^{21}$ Mycobacterium tuberculosis is transmitted only through air containing microdroplets of $\mathrm{TB}$ bacteria and is not transmitted by touching hard surfaces such as bed linen, etc. ${ }^{21}$

Ebola does not occur in Australia but given the international coverage of ongoing outbreaks in Africa, there is an expectation that students should know that full PPE protection was needed. Of more relevance to Australia, it is of concern that knowledge of the importance of using gloves, mask and gowns for patients with MRSA was not $100 \%$. This gap in the students' knowledge is of concern because MRSA is a global infection control problem in health facilities with a high mortality rate. ${ }^{23}$ The use of appropriate hand hygiene and PPE is an essential strategy to control the spread of MRSA in health facilities. ${ }^{24}$ These gaps appear to be primarily gaps in knowledge and may indicate a lack of training in the course specifically regarding principles of the use of PPE.

There appears to be good understanding of best practice with sharps disposal with the vast majority of students indicating they always disposed used needles in the sharps disposal container and would always report a sharps injury to their supervisor. However, some students did admit that at times they were recapping needles after use and a few indicated that at times they were bending used needles. Students in this study have indicated that although seniors teach them not to recap used needles, they have observed seniors doing otherwise in practice. The Australian Guidelines for the Prevention and Control of Infection in Healthcare clearly states that needles must not be recapped, bent or broken after use.

The rates of immunisation of students is adequate. Interestingly nearly two-third students reported having received the BCG vaccine which is not part of the national immunisation policy in Australia nor is it recommended for medical students. This may be because a large proportion of medical students are international students from Asian countries or domestic students who were born overseas where BCG vaccination is part of national immunisation programs. Given that the incidence of TB is very low in Australia (TB case notification rate of 5.5 cases per 100,000 population), ${ }^{25}$ it is perhaps not surprising that few students indicated that they did not know if annual tuberculin testing was indicated for medical students studying in Australia. The current policy recommends regular TB screening only for health care workers working in certain settings with an increased risk of exposure to TB.

Despite the limitations, this study has identified important gaps in the current knowledge and practice of standard precautions. To our knowledge this was the first study conducted among medical students to examine their knowledge and practices of standard precautions as a whole. The findings of the present study could be employed to design interventions which could upskill students' knowledge of the appropriate infection control and prevention strategies. Future research could investigate the effect of promoting this knowledge among medical students.

\section{LIMITATION OF STUDY}

This study has some limitations. This was a pilot study only, using a convenient sample of 50 students. As this was not a random sample there may be sampling bias. Students may be subject to recall error and social desirability bias. To minimize these self-reported biases, some strategies were used during the data collection. At the start of the questionnaire, we confirmed anonymity to the participants and reiterated the aim of the research which was to identify gaps in students' knowledge and practice of standard precautions. Therefore, the information collected in the questionnaire would be useful for designing approaches to develop these aspects in students. Moreover, students were asked to report on the routine and frequent practices.

\section{CONCLUSION}

The knowledge and practice of standard precautions among Australian medical students was not adequate. There was evidence that some practices of standard precautions are deteriorating in senior medical students compared to junior students. This can be related to the poor practices of senior staff. There were some major gaps in the knowledge of appropriate PPE for serious infections like serious respiratory viruses, TB, and MDRSA. There is a need for standardized and regular student training in the use of standard precautions in infection prevention and control.

\section{Conflict of Interest: None.}

\section{Authors' Contribution}

MPI: Direct contribution, KU: Intellectual contribution, MSI: Intellectual contribution, VJ: Intellectual contribution, $\mathrm{JH}$ : 
Intellectual contribution, AAC: Intellectual contribution, NUSK: Intellectual contribution.

\section{REFERENCES}

1. World Health Organization. WHO Guidelines on Hand Hygiene in Health Care. 2009. Avaialabe at: https:/ /www.who.int/gpsc/ 5may/tools/who_guidelines-handhygiene_summary.pdf (Accessed on December 12, 2020)

2. NHMRC. Australian Guidelines for the Prevention and Control of Infection in Healthcare. 2019. [Internet] Avaialabe at: https:// www.nhmrc.gov.au/about-us/publications/australianguidelines-prevention-and-control-infection-healthcare-2019 (Accessed on December 12, 2020)

3. Allegranzi B, Pittet D. Role of hand hygiene in healthcareassociated infection prevention. J Hosp Infect 2009; 73(4): 305315.

4. Gammon J, Morgan-Samuel H, Gould D. A review of the evidence for suboptimal compliance of healthcare practitioners to standard/universal infection control precautions. J Clin Nurs 2008; 17(2): 157-167.

5. Askarian M, McLaws ML, Meylan M. Knowledge, attitude, and practices related to standard precautions of surgeons and physicians in university-affiliated hospitals of Shiraz, Iran. Int J Infect Dis 2007; 11(3): 213-219.

6. Pittet D, Simon A, Hugennot S, Pessoa-Silva CL, Sauvan V. Hand hygiene among physicians: performance, beliefs and perceptions. Ann Int Med 2004; 141(1): 1-8.

7. Amin TT, Al Noaim KI, Bu Saad MA, Al Malhm TA, Al Mulhim AA, Al Awas MA. Standard precautions and infection control, medical students' knowledge and behavior at a Saudi university: the need for change. Glob J Health Sci 2013; 5(4): 114-125.

8. Tavolacci MP, Ladner J, Bailly L, Merle V, Pitrou I, Czernichow P. Prevention of nosocomial infection and standard precautions: knowledge and source of information among healthcare students. Infect Control Hosp Epidemiol 2008; 29(7): 642-627.

9. Colosi A, Ergasti G, Murzilli G, Paolini V, Semeraro V, Trapini $\mathrm{M}$, et al. Healthcare students and their knowledge of healthcare infections. Ann di Igiene 2011; 23(3): 203-208.

10. Mann CM, Wood A. How much do medical students know about infection control?. J Hosp Infect 2006; 64(4): 366-370.

11. Kulkarni V, Papanna MK, Mohanty U, Ranjan R, Neelima V, Kumar N, et al. Awareness of medical students in a medical college in Mangalore, Karnataka, India concerning infection prevention practices. J Infect Public Health 2013; 6(4): 261-268.

12. O'Brien D, Richards J, Walton KE, Phillips MG, Humphreys H. Survey of teaching/learning of healthcare-associated infections in UK and Irish medical schools. J Hosp Infect 2009; 73(2): $171-175$.
13. Kaur R, Razee H, Seale H. Exploring the approaches used to teach concepts of hand hygiene to Australian medical students. J Infect Prev 2015; 16(4): 162-166.

14. Kaur R, Razee H, Seale H. Facilitators and barriers around teaching concepts of hand hygiene to undergraduate medical students. J Hosp Infect 2014; 88(1): 28-33.

15. Sax H, Allegranzi B, Uçkay I, Larson E, Boyce J, Pittet D. 'My five moments for hand hygiene': a user-centred design approach to understand, train, monitor and report hand hygiene. J Hosp Infect 2007; 67(1): 9-21.

16. Australian Commission on Quality and Safety in Health Care. HHA Manual. 2018, [Internet] Avalible from: https://www. safetyandquality.gov.au/

17. Cresswell P, Monrouxe LV. 'And you'll suddenly realise 'I've not washed my hands': medical students', junior doctors' and medical educators' narratives of hygiene behaviours. BMJ Open 2018; 8(3): e018156.

18. Barroso V, Caceres W, Loftus P, Evans KH. Hand hygiene of medical students and resident physicians: predictors of attitudes and behaviour. Postgrad Med J 2016; 92(1091): 497-500.

19. MacIntyre CR, Chughtai AA. Facemasks for the prevention of infection in healthcare and community settings. Br Med J 2015; 350(1): h694.

20. Australian Government-The Department of Health. Interim advice on non-inpatient care of persons with suspected or confirmed Coronavirus disease (COVID19), including use of personal protective equipment (PPE). 2020. Available at: https://www. health.gov.au/sites/default/files/documents/2020/03/interim (Accessed on December 22, 2020)

21. Australian Government-The Department of Health. Infection control guidelines for the management of patients with suspected or confirmed pulmonary tuberculosis in healthcare settings. 2016. Availabe at: https://www1.health.gov.au /internet/main/ publishing.nsf/Content/cda-cdi4003i.htm (Accessed on Dec 22, 2020)

22. Kennedy S. The new face of an old disease: TB over 3 decades. Infect Dis Childr 2017; 30(2): 1.

23. Andersen BM. MRSA Prevention. Prevention and control of infections in hospitals: Springer; 2019 [Internet] Available at: https:// doi.org/10.1007/978-3-319-99921-0

24. Lambert A. Does the use of personal protective equipment (PPE) while examining patients with methicillin-resistant Staphylococcus aureus (MRSA) decrease the incidence of nosocomial transmission in the hospital setting? Evid Base Pract 2017; 20(6): 14.

25. Viney Kerri. The Australian tuberculosis risk factor survey. 2019, [Internet] Available at: https://rsph.anu.edu.au/research/ projects/australian-tuberculosis-risk-factor-survey (Accessed on December 22, 2020) 